\title{
Chimeric Antigen Receptor T-cell Therapy for Relapsed or Refractory Large B-cell Lymphoma - Latest Data from the ZUMA-1 Study
}

\author{
An Expert Interview with Javier Munoz \\ Cancer Immunotherapy Program; Department of Lymphoma and Myeloma, Banner MD Anderson Cancer Center, Gilbert, Arizona, USA
}

DOI: https://doi.org/10.17925/EOH.2019.15.1.16

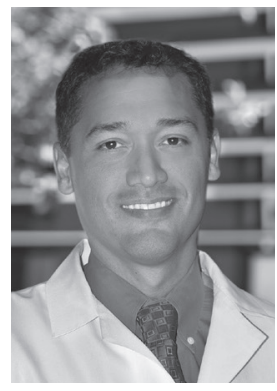

\section{Javier Munoz}

Dr Javier Munoz is an Adjunct Assistant Professor in the Department of Lymphoma/Myeloma at the Banner MD Anderson Cancer Center and Director of the Cancer Immunotherapy Program in Arizona, USA. Dr Munoz studied medicine at the Universidad Peruana Cayetano Heredia in Lima, Peru and went on to complete a fellowship in Investigational Cancer Therapeutics at the MD Anderson Cancer Center in Texas, USA. Dr Munoz now specialises in chimeric antigen receptor (CAR) T-cell therapy for the treatment of Iymphoma and is the local principal investigator at the Banner MD Anderson Cancer Center for multiple CAR T-cell trials, including ZUMA-1, ZUMA-2, ZUMA-5, ZUMA-7 and ZUMA-12. He is a member of the American Society of Clinical Oncology (ASCO) Diversity Mentoring Program, ASCO Virtual Mentoring Program, American Society of Hematology (ASH) Practice Partnership, ASH Health Volunteers Overseas Program, and the ASH Minority Resident Program as a mentor. Dr Munoz has published his research in New England Journal of Medicine, Journal of Clinical Oncology, Lancet Oncology and Blood.

\section{Keywords}

ZUMA-1, CAR T-cell therapy, axicabtagene ciloleucel, large B-cell lymphoma, CD-19, ongoing response

Disclosure: Javier Munoz has a consultancy and advisory role for Genentech, Alexion, Bayer, Bristol-Meyers Squibb Juno/Celgene, Kite, Gilead, Kyowa, Merck and Pfizer. He is on the speakers' bureau participation for AstraZeneca, Kite, Gilead, Bayer and Janssen/Pharmacyclics.

Acknowledgements: Medical writing assistance was provided by Katrina Mountfort of Touch Medical Media, and supported by Touch Medical Media.

Review Process: This is an expert interview and, as such, has not undergone the journal's standard peer review process.

Compliance with Ethics: This article is an opinion piece and does not report on new clinical data, or any studies with human or animal subjects performed by any of the authors.

Authorship: The named author meets the International Committee of Medical Journal Editors (ICMJE) criteria for authorship of this manuscript, takes responsibility for the integrity of the work as a whole, and has given final approval for the version to be published.

Received: 15 February 2019

Accepted: 13 June 2019

Citation: European Oncology \& Haematology. 2019;15(1):16-7

Corresponding Author: Javier Munoz, Department of Lymphoma and Myeloma, Banner MD Anderson Cancer Center, 2946 East Banner Gateway Drive, Gilbert, AZ 85234, USA. E: javier.munoz@bannerhealth.com

Support: No funding was received in

the publication of this article. arge B-cell lymphoma includes diffuse large B-cell lymphomas, primary mediastinal B-cell lymphoma and transformed follicular lymphoma. These have historically been treated with rituximab, cyclophosphamide, doxorubicin, vincristine and prednisone (R-CHOP). High-dose salvage chemotherapy and autologous stem-cell transplantation (SCT) can be used in case of relapse; however, patients who fail R-CHOP or relapse following SCT have a bleak prognosis. ${ }^{1}$

Recently, new data from the ZUMA-1 study have been released. ${ }^{4}$ In an expert interview, Dr Munoz discusses these findings, which provide long-term efficacy and safety data for axicabtagene ciloleucel.

\section{Q. How have the latest findings of the ZUMA-1 study increased our understanding of the efficacy and safety of CAR T-cell therapy?}

The main objective of this 2-year update was to assess long-term safety and efficacy of axicabtagene ciloleucel in patients with refractory large B-cell lymphoma. ${ }^{4}$ At a median follow up of 27.1 months, $39 \%$ of patients maintained ongoing response with axicabtagene ciloleucel. Regarding efficacy, the median overall survival has not been reached at this point. Furthermore, 93\% of patients with ongoing response at 12 months remained in response at 24 months. Regarding safety, there have been no new cases of late axicabtagene ciloleucelrelated cytokine release syndrome, neurotoxicity, secondary cancers or deaths since the 1-year follow-up. Taken together, we believe that our results support the fact that axicabtagene ciloleucel both safely and effectively induces durable responses in patients with large B-cell lymphoma who otherwise lack curative options.

\section{Q. What were the limitations of this study?}

The study was mainly designed to show an improvement in response, and we did not assess other variables, such as the effect of axicabtagene ciloleucel on quality of life. Data for immunoglobulin levels were not available because collection of immunoglobulin concentrations was decided by each investigator instead of a protocol-specific mandatory assessment. Having said that, we do know that during the course of the study only $31 \%$ of patients received 
intravenous immunoglobulin. Thus, only a minority of patients require immunoglobulin replacement from a practical standpoint.

Another possible limitation would be lack of even more prolonged follow-up beyond 2 years. However, results from large retrospective studies of lymphoma survivorship databases suggest that sustained responses 24 months after chemoimmunotherapy were associated with improved overall survival at 5 years in patients with diffuse large B-cell lymphoma who previously received first-line treatment or after autologous SCT. ${ }^{5}$

\section{Q. Which patients are most likely to respond to this therapeutic approach?}

The ZUMA-1 study led to the approval of axicabtagene ciloleucel in the by the US Food and Drug Administration and European Medicines Agency for the treatment of patients with relapsed or refractory large B-cell lymphoma that received two or more prior systemic therapies. It allowed enrolment of patients with diffuse large B-cell lymphoma, primary mediastinal B-cell lymphoma, and transformed follicular lymphoma that had no response to their last chemotherapy and/or relapse within 12 months of autologous SCT.

A subgroup analysis was performed on patients with double-expressor or high-grade B-cell lymphoma (including double- or triple-hit lymphoma) and showed $48 \%$ of patients in ongoing response. ${ }^{6}$ Currently ongoing clinical trials will help us elucidate other subgroups of patients that may benefit from this therapeutic strategy (ZUMA-12; ClinicalTrials.gov Identifier: NCT03761056).

\section{Q. What factors affect the development of resistance, and how can we best address this challenge?}

Potential mechanisms of resistance include antigen escape (CD19 loss) and programmed cell death ligand 1 (PD-L1) expression. Relapses may occur when CD19-negative clones thrive even though obliteration of CD19-positive cells has successfully occurred after infusion of CAR $T$ cells. There has been some discussion regarding persistence of CAR T cells as a factor for resistance; however, in the ZUMA-1 study, it was hypothesised that functional CAR T-cell persistence may not be required for long-term remission. This suggestion is based on evidence of B-cell recovery and decreasing detectable gene-marked CAR T cells despite the fact that these patients remain in ongoing remission.

\section{Q. What are the next steps in the clinical development of axicabtagene ciloleucel?}

The next steps would be to test axicabtagene ciloleucel in other CD19-expressing Iymphomas (e.g. ZUMA-5; ClinicalTrials.gov Identifier: NCT03105336) $)^{8}$ or in earlier therapeutic settings (e.g. ZUMA-7; ClinicalTrials.gov Identifer: NCT03391466) ${ }^{9}$ while finding ways to improve efficacy and decrease toxicity. ZUMA-5 is testing this antiCD19 construct in indolent lymphomas like follicular and marginal zone Iymphoma. The ZUMA-7 trial is a randomisation between autologous stem cell transplant versus CAR T-cell therapy in first relapse. This is an exciting time to be an oncologist and I am honoured to be an investigator on these CAR T-cell studies. $\square$

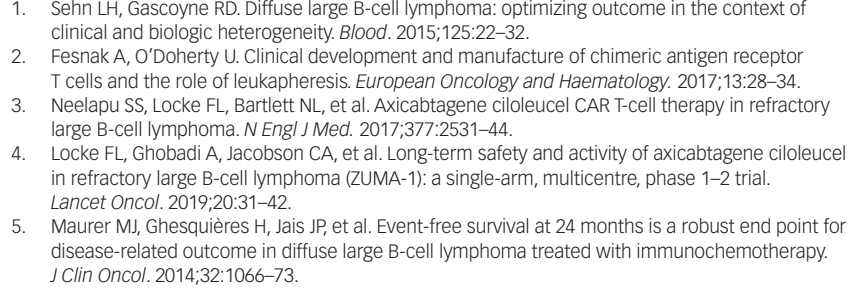

5. Maurer MJ, Ghesquières $\mathrm{H}$, Jais JP, et al. Event-free survival at 24 months is a robust end point for disease-related outcome in diffuse large B-cell lymphoma treated with immunochemotherapy. J Clin Oncol. 2014;32:1066-73.

6. Y. Jakobsen $L H$, Bøgsted $M$, Brown $P N$, et al. Minimal loss of lifetime for patients with diffuse large B-cell lymphoma in remission and event free 24 months after treatment: a Danish population-based study. J Clin Oncol. 2017;35:778-84.

7. ClinicalTrials.gov. Efficacy and safety of axicabtagene ciloleucel as first-line therapy in participants with high-risk large B-cell Iymphoma (ZUMA-12). ClinicalTrials.gov Identifier: NCT03761056. Available at: https://clinicaltrials.gov/ct2/show/NCT03761056 (accessed 20 June 2019).

8. ClinicalTrials.gov. A phase 2 multicenter study of axicabtagene ciloleucel in subjects with relapsed/ refractory indolent non-Hodgkin lymphoma (ZUMA-5). ClinicalTrials.gov Identifier: NCT03105336. Available at: $h$ ttps://clinicaltrials.gov/ct2/show/NCT03105336 (accessed 4 March 2019).

9. ClinicalTrials.gov. Efficacy of axicabtagene ciloleucel compared to standard of care therapy in subjects with relapsed/refractory fiffuse large B-cell Iymphoma (ZUMA-7). ClinicalTrials. gov Identifier. NCT03391466. Available at: https:///linicaltrials.gov/ct2/show/NCT03391466 (accessed 4 March 2019). 